\title{
El programa fundacionalista del saber en la enciclopedia de las ciencias filosóficas
}

\author{
Hegel and the foundationalism of \\ the Encyclopedia of Philosophical Sciences
}

\author{
JUAN J. PADIAL BENTICUAGA \\ Universidad de Málaga (España)
}

Recibido: 12-04-2014 Aceptado definitivamente: 24-05-2014

\section{RESUMEN}

Este artículo trata del programa de la Enciclopedia de las ciencias filosóficas de Hegel. Programa que Hegel delinea en la introducción a dicha obra, y que culmina en el silogismo de silogismos que concluye su obra. Desde su comienzo y término cabe advertir (1) la diferencia de la Enciclopedia hegeliana con las enciclopedias dieciochescas, y (2) la relación de la ciencia filosófica hegeliana con lo empírico.

$$
\begin{gathered}
\text { PALABRAS CLAVE } \\
\text { HEGEL, ENCICLOPEDIA, FUNDACIONALISMO, CIENCIA }
\end{gathered}
$$

\footnotetext{
ABSTRACT

This paper focus on the Hegelian program of the Encyclopedia of the Philosophical Sciences. Hegel explains his program in the "Introduction", and in the last pages of the Encyclopedia, the syllogism of syllogisms. From its beginning and from its end, it is possible to notice (1) the difference between the Encyclopedia and other encyclopedias of the eighteenth-century, like the French one or the British one; and (2) the relationship of the Hegelian notion of philosophical science with the empirical.

KEY WORDS

HEGEL, ENCYCLOPEDIA, FOUNDATIONALISM, SCIENCE
} 


\section{EL PROGRAMA FUNDACIONALISTA DEL SABER Y LAS ENCICLOPEDIAS MODERNAS}

LA ENCICLOPEDIA DE LAS CIENCIAS FILOSÓFICAS de Hegel no está estructurada como una enciclopedia al uso. La organización de las enciclopedias suele obedecer a criterios pragmáticos, no teóricos. Responden a la necesidad de encontrar fácil y rápidamente conocimientos científicos. Por ello los editores de enciclopedias suelen adoptar como criterio organizativo el alfabético, y como modelo el Diccionario Universal de las Ciencias y las Artes que Ephraim Chambers había publicado en Inglaterra en 1728, un diccionario conocido como Cyclopaedia. ${ }^{1}$

Chambers denominó a su obra Cyclopaedia porque los artículos estaban conectados entre sí, se remitían unos a otros. Había cierta circularidad — en las doctrinas expuestas - $\pi \alpha 1 \delta \varepsilon i ́ \alpha-$. Además, proporcionaba una tabla de contenidos y un directorio en el que se mostraba el orden y disposición de las voces. Este directorio es «la mayor contribución» de Chambers a su enciclope$\mathrm{dia}^{2}$, el intento de realizar una guía en la que aparezcan las relaciones, filiaciones, y múltiples referencias cruzadas entre los diversos hallazgos científicos. Así, la Cyclopaedia venía a rehabilitar la noción griega de en kuklôi paideuein. ${ }^{3}$

Las enciclopedias pretenden instituir el ideal moderno del árbol de las ciencias. Una pretensión operativa en Bacon ${ }^{4}$ y Descartes, ${ }^{5}$ y que constituye uno de los ingredientes fundacionales de la edad Moderna. Usar la metáfora del árbol sugiere la integración de todas las ciencias en un organismo. Pero la unidad de aquellas primeras enciclopedias es problemática. ${ }^{6}$ El saber en estas enciclope-

1 Puede consultarse digitalmente en http://artfl-project.uchicago.edu/content/chamberscyclopaedia

2 Cfr.: Yeo, R., «Classifying the Sciencies» en The Cambridge History of Science, vol. 4, Eighteenth-Century Sciencie, Cambridge University Press, Cambridge Mass., p 260.

3 Cfr.: Bignami, L., «Il concetto di enciclopedia,» en Filosofia e scienze filosofiche nell' «Enciclopedia» hegeliana del 1817, F. Chiereghin (ed.), Trento 1995, 23-61. Citado por Ferrarin, A., Hegel and Aristotle, Cambridge University Press, Cambridge, Mass., p. 59.

4 Bacon, Fr., «A Description of the Intellectual Globe» en The Works of Francis Bacon, collected and edited by James Spedding, Robert Leslie Ellis, and Douglas Denon Heath, 14 vols. Longman, Londres, 1857-74. Reimpresión en Fromman, Stuttgart-Bad Cannstatt, 196163 , vol. 5 , pp. 503-04.

5 Cfr.: Descartes, R., «Meditations et principes : traduction française par L.C. d'A., duc de Luynes» en Oeuvres de Descartes publiées par Charles Adam \& Paul Tannery sous les auspices du Minstère de l'instruction publique, AT IX, 2, 14-15.

6 Para Hegel también lo es la unidad de L'Encyclopédie ou Dictionnaire raisonné des sciences, des arts et des métiers editada entre 1751 y 1772 por Denis Diderot y Jean d'Alembert. Estos editores compilaron 76.242 artículos escritos por 134 autores, que se reunieron en 17 volúmenes. Esta enciclopedia intenta organizar sistemáticamente el saber, reduciendo las ciencias a tres facultades (memoria, razón e imaginación. En esto los editores siguen el criterio de Bacon) que actuarían como órganos del entendimiento humano. Así la 
dias se organiza en ramas que se bifurcan de un tronco. En las raíces nos encontramos con la ciencia de los principios del saber, la metafísica en su versión cartesiana. El tronco estaría formado por la física, las ramas por los demás saberes, que conforman un todo indivisible con su tronco y raíces. Para los modernos, el principio - la raíz - lo es en tanto que legitima el saber, en tanto que confiere evidencia a lo sabido. El principio no es lo evidente, sino la evidencia. Aquel saber primero, que funda lo evidente, será para Bacon y Descartes la «ciencia universal»o Philosophia prima, y su objeto será el comienzo del saber, de la evidencia.

Aquí subyacen varios problemas. Uno de ellos subyace a la relación de fundamentación de la philosophia prima respecto de los demás saberes. Y está expresado en las metáforas empleadas para tratar de la relación entre el saber fundamental y los saberes fundamentados. Estas metáforas son por ejemplo el árbol del saber (Porfirio, Descartes), o el edificio del saber (como supone Kant al hablar de la arquitectónica del saber). El problema reside en que los géneros son eternos, según Platón. Y las ciencias tendrían una esencia y unos objetos definidos sub specie aeternitatis. Entre ellas cabría establecer relaciones a priori. Quedaría así descartado el problema de la historicidad del saber, en general y de las ciencias en particular.

La pretensión de los enciclopedistas del siglo XVIII de instituir el árbol de las ciencias se entronca pues en el proyecto de un programa fundacionalista del conocimiento, del saber. Esta fundamentación, recibe en las enciclopedias dieciochescas la forma de la reducción de unos saberes a otros que se consideran como fundamentales. Que el contenido de una ciencia pueda ser reducido a otra, implica considerar las ciencias como ideas platónicas. Las más generales rigen sobre sus subconjuntos. Hay una comunidad - koinonia- y unas relaciones jerárquicas entre todo lo eidético. Así, por ejemplo, Chambers dirá en unas de sus entradas que «el doctor Friend ha reducido la Química al Newtonianismo, y ha dado razón de las operaciones desde principios mecánicos». ${ }^{7}$ Parece que la reducción tiene que ver con la noción de «lo común». Lo común, en este caso, a la mecánica clásica y a la química, es lo que permite tratar mecánicamente la química. Es decir, la reducción y fundamentación de las ciencias consiste en la aplicación - Anwendung, dirá Kant- de leyes de una ciencia fundamental al contenido - las ideas generales - de otras ciencias. Esta aplicación es propia de una operación intelectual, la operación de juzgar — según Kant-, y es intrínseca al modo de generalizar - mediante los juicios determinantes y reflexivoscon que se ejerce el quehacer científico moderno, como veremos.

historia, sagrada, eclesiástica, civil y natural corresponde e incumbe a la memoria. La filosofía a la razón, y la poesía a la imaginación. Cfr., http://encyclopedie.uchicago.edu/

7 Chambers, Cyclopaedia, voz «Chymistry», vol. I. Citado por Yeo, R., op. Cit. p. 260. 
Pero la actividad científica no es la actividad del ilustrado que difunde la luz de la razón. Los enciclopedistas del siglo XVIII, aún usando la metáfora del árbol de las ciencias, sustituyen el organismo por el mapa. Bacon, al que aquellos seguían, tenía clara advertencia de ello. Por eso titula su obra «una descripción del orbe intelectual». Sólo cabe realizar una cartografía a posteriori. El orbe del saber aparece ya ahí, antecediendo a la mirada de quien lo explora. El saber aparece ya hecho, realizado por la actividad experimental e intelectual de los hombres de ciencia. El programa fundacionalista de las enciclopedias ilustradas confunde la actividad con el resultado de la actividad, como mostró Ryle. ${ }^{8}$ El resultado es el saber ya obtenido, sobre el que versa la cartografía del compilador. La actividad científica es otra y previa a la actividad cartográfica. La consecuencia de advertir esta distinción será el temprano abandono del carácter orgánico o sistemático del conocimiento. El árbol de las ciencias se diseminará en el rico y variado bosque del saber. Una arboleda formada por las diversas ciencias emancipadas de la prima philosophia. Ya en el siglo XIX, la Enciclopedia Británica tan sólo referirá la entrada «sistemas» a «ciencias particulares, y no a grandes doctrinas de filosofía natural o a los esquemas clasificatorios que constituían el prefacio a las enciclopedias anteriores». ${ }^{9}$

Aparece así otro problema. El de la autonomía de las diferentes disciplinas científicas. Un árbol, también el de las ciencias, es un organismo. La diversidad de sus órganos depende de un principio que lo ha generado, ha propiciado su crecimiento, y lo mantiene. No está clara la relación de basamento o principiación entre la Philosophia prima y las ciencias particulares. Más aún, estas ciencias se constituyen como «positivas» en un proceso de emancipación, de autonomización respecto de la filosofía. Más que formar parte de un organismo con la filosofía, enfatizan sus fronteras, se demarcan, independizan y autonomizan de ella. Así la aporía se hace ahora más aguda, y es que la filosofía se puede constituir como un saber del fundamento y fundamentado. Pero, al menos hasta el siglo XVIII la ciencia aparecía como un saber floreciente y abierto a un progreso indefinido. Los científicos formaban de facto una comunidad a la que

8 Cfr.: Ryle, G. El concepto de lo mental, Paidós, Buenos Aires, 1967. Citado por Arregui, J. Op. Cit., p. 16.

9 Yeo, R., op. Cit. p., 266. Esta consideración margina lo histórico y pragmático del saber, como si la química no tuviese una historia, y lo desarrollado por los alquímicos en las edades Antigua y Media no fueran propiamente química. Una ciencia no es un objeto científico, sino una tarea histórica y colectiva del ser humano. El punto de vista de los enciclopedistas puede considerarse como un platonismo, o como una hipostatización de la ciencia. "Las ciencias aparecen como realidades objetivas, como cuasi sustancias, que cumplen en sí mismas las propiedades que buscan descubrir en sus objetos. (...) Pero, el problema comienza cuando se predican de las ciencias las propiedades de sus objetos y se comienza a considerar que no sólo el uranio sino también la química o la filosofía tienen una naturaleza y una esencia, de manera que podamos hablar de una «auténtica medicina» o de una «verdadera filosofía».» Cfr.: Arregui, J., La pluralidad de la razón, Síntesis, Madrid, 2004, pp. 153-154. 
contribuían incesantemente. El contraste entre un saber fundado (el filosófico) pero no abierto al progreso, sino cerrado en sí mismo - excluyente de otros sistemas, por tanto-, y un nuevo saber, que se desarrolla y va conquistando nuevas y nuevas metas, pero del que se desconoce su fundamento, es bien agudo. Este contraste se agudizará cuando Kant señale que algunos de sus fundamentos, como el espacio y el tiempo, no son racionales (como sí lo son las categorías o conceptos puros).

\section{LOS MAPAS DEL SABER Y EL ÁRBOL DE LA CIENCIAS}

La concepción hegeliana de la Enciclopedia intenta fundar orgánicamente el saber, y así remedar la insuficiencia que advierte en los intentos enciclopédicos anteriores a él. Los enciclopedistas atendían a la relación que guardan unos saberes con otros. Pero aún remitiendo las voces entre sí, las entradas son exteriores unas a otras. Aquellas enciclopedias estaban construidas desde elementos atómicos-las diversas voces-, y podían reducirse a esos átomos.

Pero un organismo no es susceptible de análisis. El hecho de que cualquier voz, o entrada de una enciclopedia, sea inteligible al margen de las demás, implica que esa parte no es un miembro u órgano del todo. Igual que fuera del organismo el ojo no ve, tampoco al margen del sistema del saber, una parte del mismo es inteligible. Si lo fuese, y pudiese ser entendida con independencia, si tuviese sentido separada, significaría que no forma parte de tal organismo, que es extrínseca al sistema.

En un organismo, como Kant puso de manifiesto, «la conexión de las causas eficientes puede considerarse al mismo tiempo como un efecto por causas finales»». ${ }^{10}$ Es decir, la ordenación y disposición no es extrínseca ni a las partes ni al todo. No es algo llevado a cabo desde fuera, por un compilador, sino que la actualización del organismo es la causa de la disposición de las partes. Y a su vez, sin esta disposición el organismo no sería posible; esta disposición de sus miembros causa la misma existencia y mantenimiento del todo.

Por ello subrayará Hegel ya desde el comienzo de la Fenomenología, que «la verdadera figura en que existe la verdad no puede ser sino el sistema científico de ella». ${ }^{11}$ Esto implica que el sistema funciona como causa final de cada una de sus partes. Por ello también dirá Hegel que «lo verdadero es el todo». ${ }^{12} \mathrm{Y}$ sólo

10 Citado por Frank, M., El Dios venidero. Lecciones sobre la Nueva Mitología, Ediciones del Serbal, Madrid, 1994, p. 166.

11 Hegel, G.W.F., Fenomenología del espíritu, Fondo Cultura Económica, MéxicoMadrid, 1966. p. 9.

12 «Das Wahre ist das Ganze. Das Ganze aber ist nur das durch seine Entwicklung sich vollendende Wesen. Es ist von dem Absoluten zu sagen, dass es wesentlich Resultat, dass es erst am Ende das ist, was es in Wahrheit ist; und hierin eben besteht seine Natur, Wirkliches, Subjekt oder Sichselbstwerden zu sein.» Fenomenología del espiritu, p. 16. 
en función de su contribución al todo, al sistema u organismo del saber, pueden ser entendidas las partes o miembros. La idea hegeliana de totalidad -Ganzesimplica la rehabilitación de la teleología, de una esencia que se completa y automantiene mediante su desarrollo - Entwicklung - ${ }^{13}$

El todo, el organismo, lo absoluto, es ante todo resultado, ${ }^{14}$ un realizarse y determinarse, ya sea en la esfera ideal - la cartesiana prima philosophia - ya en las esferas reales de la naturaleza y el espíritu (las ramas del árbol según Descartes). A diferencia del acontecer histórico en las ciencias positivas, el árbol de la ciencia hegeliano es un resultado que no se separa del proceso que lo produce, ni se independiza de él, dando lugar a ciencias autosuficientes, desgajadas de su tronco y con nuevas raíces. Por ello las tres ciencias filosóficas forman parte de un solo árbol, la Enciclopedia de las Ciencias Filosóficas, un organismo en que se remiten circularmente entre sí las diferentes formas en que se realiza la Idea.

En el momento en que la Enciclopedia Británica está renunciando a la idea de sistema total, Hegel lo está rehabilitando y ofreciendo una versión alternativa del mismo. Una explicación en que se co-implican totalidad y desarrollo teleológico. El todo está en cada uno de los momentos de su realización. Estos momentos están incluidos en la eternidad del todo, que es como su acto primero. ${ }^{15}$ Como Charles Taylor ha expuesto, «la explicación teleológica es una explicación desde el punto de vista de la totalidad. Los procesos parciales son explicados desde su función en el todo». ${ }^{16}$

Cabría aducir que el compilador de una enciclopedia dieciochesca también tiene a la vista un todo. El editor intenta fundamentar unos saberes en otros, los refiere entre sí. Pero el conjunto adquiere la forma de un agregado, no de un todo que se ha desarrollado desde un único principio, sino de un conjunto de partes que interaccionan, pero no orgánicamente, sino al modo en que lo hacen las partes de un mecanismo o un conjunto de átomos, iones o moléculas. No se trata de una mera mezcla, sino de un cuerpo lógico que puede ser denominado mecanismo o quimismo. Cada parte tiene sus propios principios, que se unen o adicionan a los de otras partes que se encuentran anejas. Por ello, el conjunto de voces se presenta en la «forma de una secuencia contingente y de algo así como una mera diversidad de los principios y de las realizaciones de estos principios». ${ }^{17}$ Esta apreciación hegeliana expone un motivo fundamental de su filoso-

13 Íbidem.

14 Íbidem.

15 Por eso el proceso del saber es caracterizado como un desarrollo, no como una evolución que diese lugar a nuevos organismos autosuficientes, o como una emanación que diese lugar a novedades degradadas, imperfectas y heterogéneas. Cfr.: Ferrini, C., «Hegel on Nature and Spirit: Some systematic Remarks», en

http://www.academia.edu/1491976/Hegel_on_Nature_and_Spirit, p. 8.

16 Taylor, Ch., Hegel, Cambridge University Press, Cambridge, 1975, pp. 321-22.

17 Hegel, Enz., § 13. 
$\operatorname{far}^{18}$. La historia de la filosofía hegeliana es filosofía, y la Enciclopedia hegeliana es ciencia, no una mera exposición en que unos temas remitan en cierto modo a otros. La Enciclopedia, expone según Hegel, el manifestarse en su totalidad de la Idea. Obedece a un único principio que se desarrolla y se expone a sí mismo.

Eso no se puede decir de una enciclopedia al uso, en la que la ciencia es lo expuesto, no la exposición. Por eso las referencias circulares en las enciclopedias del siglo XVIII eran extrínsecas a unos saberes que se generaban y consolidaban mediante su especialización, es decir, en el establecimiento de fronteras. Lo expuesto por cada uno de los contribuyentes a L'Encyclopédie era científico. Las numerosas relaciones entre unas materias y otras no eran realizadas según el método de la nueva ciencia, sino desde el observador que guía al lector, exponiendo los resultados de la ciencia, y no haciendo ciencia. Pronto se advirtió la confusión entre resultado y actividad, y por eso los editores decimonónicos de la Britannica expondrán una cláusula restrictiva: «sería extremadamente afortunado para la ciencia que todas sus divisiones fuesen definidas con precisión, y que cada una se restrinja a su propia esfera». ${ }^{19}$ Aquí aparece la renuncia a una Idea que se desarrolla unitariamente en diferentes esferas y se manifiesta en diversas formas. Más que una idea germinal, rectora y primera, la Britannica expone el orbe del saber como obedeciendo a una pluralidad irreductible de principios.

\section{LA UNIDAD DEL SABER}

En cambio, según Hegel, las diversas ciencias filosóficas no tienen principios propios, separados del sistema. Ni la exposición sistemática es la ordenación contingente de diversos principios y sus realizaciones. Las diversas partes del sistema «son solamente ramas de uno y el mismo todo», ${ }^{20}$ dirá Hegel prosiguiendo con la metáfora cartesiana del árbol de la ciencia. Por eso, el sistema filosófico — científico - no es un mero agregado de disciplinas. En terminología aristotélica es holon y no pan. En el alemán de Hegel, la Enciclopedia es la ciencia que expone el Ganzes, el orden y conexión total del saber, y en tanto que éste es su objeto, es científica, o mejor, es la Ciencia.

Que la filosofía sea la exposición de una totalidad, de un Ganzes, de un holon implica que es indivisible, tan indivisible como lo es una sustancia natural. No en vano, Hegel se refiere a la metáfora del árbol, y no de las ciencias, sino de la ciencia. Según Aristóteles, la sustancia es ho àn tode ti òn kaì chôristòn êi, «siendo algo determinado es también capaz de existencia separa-

18 No es una tesis que tenga sólo validez para su tratamiento de la historia de la filosofía, aunque el contexto en el que está formulada sea el de la fundamentación filosófica de la historia de la filosofía.

19 Encyclopaedia Britannica, $3^{\text {a }}$ edición, vol., 14, p. 665. Citado por Yeo, R., «Classifying the Sciencies», op. Cit. p. 266.

20 Hegel, Enz., § 13.

Suplemento 19 (2014) de Contrastes. Revista internacional de filosofía 
da». ${ }^{21}$ También el sistema es así, es separado, no necesita ni remite a nada fuera de sí para ser pensado. Es inteligible en sí; sin tomar su método de una ciencia externa, como la matemática, que presta el carácter de ciencia rigurosa a múltiples ciencias empíricas. Tampoco remite a la observación y experimentación, como si la fuente de la verdad residiese en lo empírico. Aquí aparece una profunda diferencia con el proyecto fundacionalista de los enciclopedistas dieciochescos. Y es el principio de las ciencias empíricas, para ellos, debería provenir de la philosophia prima. Aquellas tomarían sus principios de la evidencia averiguada por la filosofía. En Hegel, en cambio, no se da un trasvase de la filosofía a las ciencias empíricas, sino al contrario, una reflexión filosófica sobre lo logrado por las ciencias empíricas. La filosofía no sale de sí, las ciencias reales son filosóficas: la filosofía de la naturaleza y del espíritu. Las ramas del árbol no se escinden porque tienen la misma naturaleza (filosófica) que el tronco.

Es evidente que la ciencia hegeliana no es una ciencia empírica. Pero conviene averiguar el por qué, no meramente constatarlo. Kant había fundamentado el conocimiento de los objetos de experiencia, limitando el conocimiento humano al conocimiento empírico, ateniéndose o reduciéndose a él. Para Kant todo objeto posible es el fruto de una síntesis. Para Hegel, en cambio, el sistema de la ciencia es un todo, pero no una síntesis. En una síntesis, la actividad del intelecto se diferencia del contenido unificado. Lo dado empíricamente - empirisch gegeben - y lo unificante se diferencian, y es preciso considerarlos separadamente. En el tratamiento kantiano del conocimiento cabe discernir elementos formales y materiales. Si cabe sintetizar es porque lo dado carece de unidad intrínseca. Por eso, la actividad sintética es para Kant una actividad constructiva. Se construye un posible objeto de experiencia mediante la conexión Verbindung - de lo múltiple dado. Por ello, lo que se construye es la unidad del objeto de experiencia, una unidad a priori, con la que no cuenta lo múltiple y heterogéneo - das Mannigfaltige - dado a los sentidos.

La dualidad «esquema o forma», y «contenido material, empírico, informe, necesitado de organización» es - como puso de relieve Donald Davidson- uno de los dogmas del empirismo. ${ }^{22}$ Este depender y esta dualidad entre lo formalintelectual y lo material-empírico — der «Stoff» der Anschauung—, consagra la mutua exterioridad entre lo posible y lo real, entre lo pensable y lo cognoscible. Lo real aparece como lo que precisa y aguarda la organización que le prestará el esquema categorial, como lo Mannigfaltige, la heterogeneidad y pluralidad pura, lo disperso y fraccionado infinitamente. Aparece así, la actividad cognoscitiva, no ya sólo como la construcción de la unidad del objeto, sino como una pretensión desconsiderada para con lo real. Esta pretensión lleva a la ecuación «real =

21 Aristóteles, Met., $\Delta 8,1017$ b 25-26.

22 Davidson, D., "On the Very Idea of a Conceptual Scheme» en Inquiries into Truth and Interpretation, Oxford University Press, Oxford, 1985, pp. 183-98. 
empírico $=$ fáctico $=$ dado $=$ cognoscible mediante las formas que impone el entendimiento $=$ existente en cuanto que se corresponde con un hecho empírico»).

Pues bien, la Enciclopedia de las ciencias filosóficas de Hegel no es pensada como un agregado de ciencias - las enciclopedias dieciochescas - ni como una síntesis filosófica de saberes empíricos, saberes heterogéneos entre sí y también respecto de la filosofía. Si fuera así, la filosofía descansaría en lo empírico, como aquello que le es dado para realizar una síntesis, irremediablemente contingente, dependiente del estado de las ciencias en un momento histórico dado. La unidad de la ciencia no es la de un objeto de experiencia. En la síntesis cognoscitiva, la forma a priori unifica lo múltiple y heterogéneo de la sensibilidad. El sistema, en cambio añade a la unificación formal, la unificación final. Por ello, la ciencia filosófica no es sólo un todo de objetos cognoscitivos, sino un todo que es causa de sí mismo, un organismo, un fin en sí y para sí mismo. Las partes mantienen el todo, y el todo constituye las partes, las genera y dispone para que lo mantengan. El arbor scientiae, la filosofía, es el único saber que reúne este requisito, por eso es la única ciencia para Hegel.

En este punto Kant y Hegel se diferencian radicalmente. Según Hegel, el saber es un todo, y eso es lo que expone el sistema. Su unidad es propia, inmanente. No es una unidad construida, puesta o impuesta a algo extrínseco, sino que la unidad del saber es intrínseca. Esto es lo que significa que lo racional, lo verdadero, es un todo, un Ganzes. Como el todo no remite fuera, se auto-fundamenta; como no es construido, lo racional o verdadero es lo primero, lo originario. Se puede expresar esto con un lenguaje ajeno a Hegel diciendo que la verdad es para él el primer transcendental.

Pero contra estas dos afirmaciones - la autofundamentación y originariedad de la verdad - se levantan inmediatamente multitud de objeciones. Quizá sea Karl Popper quien con más fuerza y éxito ha expuesto la enmienda a la totalidad que se alza inmediatamente en el realista ingenuo que todos llevamos dentro. En La sociedad abierta y sus enemigos alaba, no sin sarcasmo, a «Hegel, quien logró la más milagrosa de todas las cosas. Como un consumado lógico, era un juego de niños para su poderoso método dialéctico, extraer conejos de carne y hueso de chisteras puramente metafísicas»». ${ }^{23} \mathrm{Y}$ es que no parece que la exterioridad y multiplicidad pueda ser fácilmente exorcizada. No parece que se pueda filosofar, y mucho menos hacer ciencia, al margen de lo empírico. Nos parece, y también le parecía a Hegel, que el cosmos y los mundos culturales tienen el carácter de lo otro, respecto de lo lógico o metafísico.

Desde luego, la naturaleza tiene el carácter de realidad externa, independiente del sujeto, del que éste puede saber, al menos por la resistencia que le opone.

23 Popper, K., The Open Society and its Enemies. II. The High Tide of Phrophecy: Hegel, Marx and the Aftermath., Princeton University Press, Princeton, 1963, p. 27. 
Y la subjetividad el carácter de idealidad íntima, que cabe exteriorizar en el mundo de la segunda naturaleza, que surge de la actividad reflexiva humana, y que una vez producida parece gozar de vida propia. Así, lo que está en juego es la posibilidad misma de lo que Hegel denominó filosofía real: la de la naturaleza y la del espíritu, frente a la lógica o metafísica. Pero para resolver esta aporía hay que insistir en que la filosofia es un saber de totalidad, no un agregado de diferentes disciplinas con diversos principios.

\section{EL CÍRCULO DEL SABER}

¿Qué significa entonces que la filosofía es la exposición científica del todo? En primer lugar que los miembros del todo no son elementos autosuficientes e indiferentes entre sí, que se relacionan extrínsecamente, «sino todos organizados en el que todos los elementos que constituyen el todo juegan un papel determinado e importante». ${ }^{24}$ Tampoco las diferentes ciencias son independientes unas de otras. Como si la lógica, y las esferas reales de la naturaleza y el espíritu fuesen compartimentos estancos. El todo está presente en cada uno de los todos. Son todos ${ }^{25}$ dentro del todo. «Y porque el círculo particular es en sí mismo totalidad, rompe también los límites de su elemento y funda una esfera ulterior. ${ }^{26}$ Es por ello que la lógica traspasa a la naturaleza, cuando la Idea absoluta mira fuera de su elemento. Y por lo que la Idea retorna a sí desde la alienación en el espacio y el tiempo de la naturaleza. Las esferas aunque diferentes, no son independientes. Además, las esferas en modo alguno están constituidas de partes que pudieran preexistir al todo. Tampoco ellas mismas son al margen de él, sino que éste es su principio y aquellas son su realización. Por eso el todo es como la sustancia aristotélica, lo primero en todos los sentidos, kai logôi kai gnôsei kai chronoi. ${ }^{27}$

A ese todo que es la verdad, el Ganzes y que es lo primero en todos los sentidos, Hegel lo denomina Idea absoluta. Como Ganzes es un totalidad orgánica, un holon en el sentido aristotélico; no un pan, un mero agregado de partes independientes y autosubsistentes. Como verdad, esa totalidad orgánica tiene carácter lógico. Es el organismo de categorías lógicas, la compleción de categorías

24 Stern, R., Hegel, Kant and the structure of the object, p. 97.

25 Hegel, G.W.F., Enz., § 15.

26 Íbidem.

27 Aristóteles, Metafísica, Z 1, 1028 a $32-1028$ b 2. «Pero «primero» se dice en muchos sentidos. Pues bien, en todos ellos es primera la entidad: en cuanto a la noción, en cuanto al conocimiento y en cuanto al tiempo. En efecto, ninguna de las otras cosas que se predican es capaz de existencia separada, sino solamente ella. Y también ella es primera en cuanto a la noción (ya que en la noción de cada una (de las demás) está incluida necesariamente la de entidad); $y$, en fin, pensamos que conocemos cada cosa, sobre todo, cuando sabemos qué es el hombre o el fuego, más que si sabemos la cualidad, la cantidad o el dónde; y es que, incluso, conocemos cada una de estas cosas cuando sabemos qué es la cantidad o la cualidad.» 
racionales. «La Idea absoluta es la totalidad orgánica de todas las categorías lógicas; lo universal especulativo presente e impregnando todo. Esto significa que toda categoría ha de considerarse en una doble perspectiva: (1) como un predicado de Dios, la forma real del ser divino; (2) como un predicado del mundo que expresa el núcleo sustancial oculto de todo ser. Pero, además hay una tercera perspectiva, (3) la de la lógica misma que se centra en la necesidad inmanente de las relaciones entre las categorías, y que culmina en lo especulativamente concreto, en la Idea absoluta». ${ }^{28}$ Así, la Idea absoluta se muestra a sí misma, se presenta por sí en el mundo y en el espíritu, siendo como su núcleo racional. Es más, la posibilidad de comprender las esferas de la naturaleza y del espíritu estriba en este núcleo racional de categorías lógicas; sólo mediante la Idea cabe ciencia de lo real, cabe concepto de la naturaleza o del espíritu. En esto consiste lo nuclear del tercer silogismo con que termina la Enciclopedia de las Ciencias Filosóficas. Ella, la Idea absoluta, es la omnitudo possibilitatis de Leibniz, y por lo tanto se convierte con lo pensable, pues como había dicho Kant las condiciones de pensabilidad de un objeto, son las de su posibilidad.

Ahora podemos discernir mejor en la totalidad, porque las esferas de la Naturaleza y del Espíritu tienen en su base la de la Lógica. Se trata de una esfera de categorías que impregna a las demás esferas, que está realmente presente en las otras dos, como su sustancia. Varía la forma, pero la sustancia es la misma. La naturaleza es la Idea en la forma de su estar fuera de sí, en la exterioridad. El espíritu es la Idea en la forma de su volver a sí desde la exterioridad. La idea absoluta, es la sustancia oculta, y que se presenta a sí misma en la Ciencia filosófica. Pero la lógica no es el todo, sino una esfera del todo. Y ya había señalado Aristóteles que la sustancia es lo primero temporal, cognoscitiva y nocionalmente.

\section{EL SILOGISMO DE SILOGISMOS Y LA AUTOFUNDAMENTACIÓN DEL SABER}

Como la Idea absoluta es la totalidad orgánica de las categorías lógicas, ha de permitir pensar el tipo de organismo que es el todo. Como organismo, sus miembros se implican entre sí. La disposición de sus miembros causa la existencia del organismo, y la realización o actualización del organismo es causa de la disposición y co-existencia de los miembros. Para poder pensar así el Ganzes, Hegel usa la figura del silogismo de silogismos.

Para comprender qué sea para Hegel un silogismo conviene comprender en qué se diferencia éste del juicio. Hegel sigue a Kant en el tratamiento del juicio. El juicio no es tanto un modo de enunciar lingüísticamente como una operación intelectual. Por ello la forma «S es P», es tan sólo la expresión lingüística de la

28 Williams, R., Tragedy, Recognition and the Death of God, Oxford University Press, Oxford, 2012, pp. 237-238. 
actividad sintética realizada por el conocimiento. En el juicio, un concepto -el predicado - válido respecto de una multiplicidad de representaciones, aparece como válido respecto de la representación que está del lado del sujeto. Esta validez reside en el hecho de que el predicado es la determinación conceptual común a varias representaciones. El predicado es el concepto en que se homogeneizan diversos objetos. Así, por ejemplo en el juicio «los metales son cuerpos», «cuerpo» aparece como la nota común y que homogeneiza a los diversos metales, ya sólidos como el hierro, ya líquidos como el mercurio. Así el juicio permite «el conocimiento mediato de un objeto». ${ }^{29} \mathrm{El}$ objeto está representado en el sujeto del juicio. Es la referencia, lo dado en la intuición, y que es pensado mediante el predicado. Es indistinto que el juicio sea particular o universal. También los sujetos a los que precede un cuantificador universal se refieren a todos los $x$ que cumplen un predicado. Así el sujeto aparece como lo dado en la intuición, y el predicado como el concepto.

Pues bien, la facultad de juzgar (Urteilkraft) actúa como un puente Verbindungsmittel - entre la sensibilidad y el entendimiento, es decir entre lo dado y la facultad que proporciona reglas generales. «Si definimos el entendimiento en general como la facultad de las reglas, entonces el juicio consiste en la capacidad de subsumir bajo reglas, es decir, de distinguir si algo cae o no bajo una regla dada (casus datae legis)». ${ }^{30}$ En relación con las reglas hay que distinguir dos facultades: el entendimiento y el juicio. El entendimiento es la facultad de las reglas, la facultad que produce espontáneamente los conceptos, pero no la facultad de subsumir bajo reglas lo particular, o sea de aplicar - Anwendunglos conceptos a lo dado. De esto se encarga la facultad de juzgar, por la que se subsume lo particular como un caso de una determinada idea general - casus datae legis-. El juicio como facultad aplica a casos particulares - facta- las reglas o leyes conceptuales - legis-.

Así el sujeto, el término que está por la referencia, aparece como un $x$ tal que el predicado se dice de él. En el corazón pues, de la doctrina kantiana del juicio, lo que vuelve a aparecer es la facticidad, lo empírico dado, que es indeterminado al margen de la ley o la regla conceptual, que está separado de inteligibilidad, que la requiere en su mismo mostrarse como algo de lo que carece. Y en esto estriba la crítica de Hegel al tratamiento kantiano del juicio.

Según Hegel, el juicio es el momento de separación entre lo universal y lo singular, entre la ley y el caso sobre el que está vigente. Esta separación es la vigencia del predicado sobre su universo de discurso, o sobre los casos que caen bajo él. Esta asimetría entre el caso y el predicado es lo que Hegel estima como insuficiente en el juicio. La unión judicativa no es tal unidad, o no lo es suficientemente. Hegel acude a la etimología alemana de la palabra «juicio», Ur-Teil:

29 Kant, I., $K r V$. , A 68/B 93.

$30 \mathrm{KrV}$., A132 / B171.

Suplemento 19 (2014) de Contrastes. Revista internacional de filosofía 
partición originaria. Lo propio del juicio es la división, el análisis, el desmembramiento, la distribución de un concepto entre una multiplicidad indeterminada. Los dos elementos que componen el juicio no se unen conceptualmente, no se implican recíprocamente, sino que yacen separados el uno del otro. Esta separación aparece netamente en la diferente extensión del sujeto y del predicado. Por una parte el predicado extiende la singularidad del sujeto llevándolo a universalidad. Pero por ello mismo el predicado, al tiempo que está contenido en el sujeto, está más allá de él. Lo existente, —lo fáctico, el caso-, y el concepto están unidos abstractamente en el concepto. El juicio debería ser el lugar cognoscitivo de la verdad, pues el juicio expresa la relación de lo existente con el concepto. Pero no puede serlo en tanto que el predicado no ajusta su extensión al sujeto.

Kant había distinguido entre el modo determinante y el reflexionante de la operación de juzgar. Se trata de los dos modos en que opera la facultad de juzgar. El primer tipo de operación va desde un particular a un universal ya dado de antemano, a una regla ya conocida. Se trata del juicio determinante. Pero cabe que el juicio busque la regla universal que conviene a un sujeto particular. Este sería el modo reflexionante del juicio. Se trata de la modalidad judicativa más interesante pues en ella puede buscarse el concepto concreto de un existente. Pues bien, según Hegel, el encuentro del concepto que conviene a un existente no se logra en el juicio reflexionante, sino en el silogismo.

Para Hegel, el concepto es un todo de determinaciones, pero aún no desplegado. Estas determinaciones están implícitas en el concepto. Por ser un todo, el concepto es universal. Por estar implícitas las diferencias en el todo, el concepto es un universal simple, no concreto. Su desarrollo ha de mostrar cómo se adecúan o ajustan el concepto y el existente que determina tal concepto. Ese ajustarse ha de efectuarse sin residuo de facticidad. Sólo así el concepto puede decirse verdaderamente del objeto como su concepto. Y esto se logra en el silogismo de silogismos.

En el silogismo se encuentra la unidad entre lo particular, lo singular y lo universal. Esa es la función del término medio, expresar la unidad conceptual, la unidad entre los tres momentos del concepto. El concepto según Hegel, no es una mera abstracción, ni carece de contenido. El contenido es inseparable de la objetividad que lo determina. No es concepto una vaga indeterminación o una generalidad que se predique de todo $x$ designado por el concepto. Aquí renace agudamente la crítica a Kant, en concreto a su formalismo. Según Kant, la lógica es formal, aun cuando en su aspecto genera ${ }^{31}$ puede aplicarse también a contenidos empíricos. Ésta es la consideración habitual de las leyes científicas que determinan lo real-empírico como aquella $x$ sobre la que es vigente una idea general.

31 La lógica según Kant puede ser trascendental o general. 
Hegel se opone a este tipo de lógica, desconsiderada con el contenido, que llevaba consigo la pretensión de mathesis universalis.

«La razón lógica, si es considerada como razón formal, debe esencialmente hallarse también en la razón que tiene que operar con un contenido; y más bien todo contenido puede ser racional sólo por medio de la forma racional». ${ }^{32}$

Una razón formal que no considera los aspectos del contenido es la lógica extensional. No entra en el sentido o significado, en las propiedades que algo debe tener para ser definido de un modo u otro. En la lógica extensional, la forma se yuxtapone a su contenido. Aquí hay una sugerencia espacial, el ponerse junto a - iuxta positio - el contenido, como no-unitario con él, como exterior a él. Así lo lógico y lo real se diferencian. Pero se diferencian en tanto que lo lógico se asimila a lo formal y lo real se confunde con lo empírico, con lo particular. Esta diferencia es propia de la concepción kantiana del juicio y de la lógica. ${ }^{33}$

Kant pretende una interpretación formal de la lógica, de la inferencia o razonamiento, del silogismo:

«Si ahora el silogismo consiste en que la unidad de los extremos está puesta en él, y si, empero, esta unidad de un lado está considerada en absoluto como un particular por sí, de otro lado sólo como una relación extrínseca, y si se pone como relación esencial del silogismo la no-unidad, entonces esa razón, que es el silogismo, no contribuye a la racionalidad. ${ }^{34}$

La relación de la forma con el contenido no puede ser extrínseca. Y esto implica que la forma ha de operar con contenidos. No debe considerarlos como lo informe y fáctico, sino que ha de plegarse a ellos. Los predicados no son impuestos, sino que han de pertenecer a la realidad de modo intrínseco. A cada nivel de realidad le pertenece de modo exclusivo un tipo de predicados. En cada nivel de realidad se efectúa un tipo de predicados. Es la noción de realidad efectiva: Wirklichkeit. Lo pensable no es lo general sino lo determinado. En esta lógica lo importante deja de ser la cualidad y la cantidad del juicio. Si es afirmativo o negativo; universal, particular o singular. Lo relevante es la determinación

32 Hegel, G.W.F., WdL, SW: VI, 352; Mond.: p. 585

33 Considerar las propiedades —el contenido - es lo propio de la lógica intensional. Para construir una lógica intensional cabe recurrir a los mundos posibles en los que una determinada proposición es verdadera. Se trata de la estrategia seguida por Leibniz, es decir realizar una ontología modal. Desde Frege la lógica matemática, y la matemática misma, se ha abierto a lo intensional. Hay que recordar la controversia entre Hilbert y Frege a propósito de la distinción entre sintaxis y semántica, y el énfasis de los fregeanos en que la elección de los axiomas de una teoría ha de ser congruente con las propiedades de los objetos sobre los que trata. Así el programa fregeano coincide con la relación entre concepto y objeto propuesta por Hegel.

34 Hegel, WdL., Mond. SW: VI, 353; Mond.: 586 
del contenido. Esto es lo que Hegel denomina mediación, proceso dialéctico o lógica dialéctica. La dialéctica es la lógica que asume lo particular, y lo eleva a su peculiar racionalidad. No la que se impone o yuxtapone a lo empírico, sino la que integra en una unidad.

Pero por ello mismo, el juicio o el silogismo no son operaciones que realizan extrínsecamente quienes conocen. En esto reside la crítica de Hegel al juicio reflexionante kantiano. Tal tipo de juicio permite resolver a Kant la antinomia entre teleología y determinismo en la naturaleza. Cabe objetivar a los seres vivos postulando una conexión de su objetividad según una finalidad. Sin tal comparecencia de la causa final no sería posible concebir los vivientes. Pero tal finalidad es un postulado subjetivo, un «como sí» - als $o b$ - Aparece así la teleología como un principio externo al viviente, como una necesidad subjetiva para nuestra concepción. No es que el viviente determine desde sí mismo su objetividad. El juicio reflexionante no dice nada sobre la vida en sí misma, sino sobre nuestro conocimiento de la misma. En definitiva el juicio reflexionante es una forma de conocimiento que se impone a lo empírico. El giro copernicano sigue presente en la culminación de la obra kantiana, es el sujeto y no el objeto lo que funda la verdad

Pero Hegel no es un idealista subjetivo como Kant. En el segundo de los si$\operatorname{logismos}^{35}$ que cierran la Enciclopedia podría verse esta interpretación. Se trata del silogismo Naturaleza-Espíritu-Lógica. Cabe interpretar la actividad del espíritu como la que proporciona a la naturaleza su inteligibilidad. La verdad tendría su fundamento en la actividad del espíritu. Pero este silogismo no se rige por la lógica del ser: el devenir (que rige al primer silogismo), sino por la lógica de la esencia. «Es el silogismo de la reflexión espiritual en la idea». ${ }^{36}$ Cada uno de los tres términos se reflejan en los otros dos. Sólo así cabe pensar que el espíritu sea el mediador y no el fundamento. El espíritu reconoce en la naturaleza su sustancia oculta de categorías lógicas. El sujeto no aparece como principio del saber, como su fundamento, sino como el que reconoce la aparición de lo lógico en la naturaleza y el reflejo de la naturaleza en la lógica. Por eso en este silogismo «la ciencia aparece como un conocer subjetivo - ein subjectives Erkennen- $\gg,{ }^{37}$ como el reconocer, por parte de un sujeto, una inteligibilidad oculta.

Un silogismo es un todo objetivo, un concepto, posible por el término medio. El silogismo de silogismos permite pensar la totalidad orgánicamente, porque la triple objetividad relacionada aparece implicándose mutua y necesariamente. Y esto lo hace mediante la variación del término medio. Así es como un término medio no sólo da razón de los otros, sino que los otros dan razón de él. Como cada mediación implica conceptualmente a las otras dos, el silogismo de silo-

35 Hegel, G.W.F., Enz., § 576.

36 Ibidem.

37 Ibidem.

Suplemento 19 (2014) de Contrastes. Revista internacional de filosofía 
gismos aparece como un concepto de conceptos. Por ello dirá Hegel que «sólo mediante la naturaleza de este concluir -Zusammenschliessens -, o sea, mediante esta triplicidad de silogismos con los mismos términos, es como verdaderamente se comprende un todo con su organización». ${ }^{38}$ El silogismo de silogismos aparece así como la verdadera en kuklôi paideuein, como el circular mismo de la objetividad en el concepto, de las esferas de la ciencia en su exposición sistemática.

El primer silogismo se rige por la lógica del ser. En él se expone el tránsito - übergehen - entre las ciencias filosóficas. «Lo lógico deviene naturaleza, y la naturaleza espíritu». ${ }^{39}$ En este primer silogismo aparece la inidentidad entre Idea, Naturaleza y Espíritu. Estas tres esferas aparecen en el primer silogismo como gozando de consistencia ontológica, como entes, autónomos y separados. No cabría una ciencia sino tres ciencias filosóficas distintas, autónomas y enlazadas extrínsecamente. El orden de este silogismo es el que estructura la Enciclopedia hegeliana, que aparece por ello como la exposición de la génesis y constitución de lo lógico, su devenir a la naturaleza y el aparecer y constituirse del espíritu como vuelta a la Idea. Pero se trata de una transición externa, como la que estructuraba las relaciones y referencias de las enciclopedias dieciochescas.

El espíritu no es fundamento de la verdad. Eso lo sabemos por el segundo silogismo. Por el primero advertimos que tampoco lo es la naturaleza, lo extramental o lo empírico. Este «silogismo está [entero] en la idea y la naturaleza está esencialmente determinada sólo como punto de paso y momento negativo. ${ }^{40}$ La naturaleza no aparece como el fundamento de la verdad. Hegel no es ni empirista ni realista. Por eso todo el silogismo está en la idea, en la lógica. Pero ¿qué necesidad hay que lo lógico devenga naturaleza? Justamente la inidentidad entre lógica y espíritu. No hay según Hegel una relación intrínseca entre la lógica y la actividad subjetiva. La relación entre la lógica y el espíritu es externa.

La unidad que se da en el silogismo tiene una fuerte semejanza con la unidad de los organismos vivos. En estos, las diferentes partes materiales no son separables del principio unificador, de la actividad organizativa. Las partes conforman una totalidad en el organismo, pero esa totalidad no es posible por las partes, sino por el principio que las integra activamente. Las partes son integradas, y eso significa que su independencia y autosubsistencia es anulada, por la unidad activa y negadora del principio en que son integradas. Por ello, la realidad de una parte en el organismo es la del organismo, la del todo. Su realidad independiente ha sido cancelada por la «unidad negativa», por el principio o concep-

38 Hegel, G.W.F., Enz., § 198.

39 Hegel, G.W.F., Enz., § 575.

40 Ibidem.

Suplemento 19 (2014) de Contrastes. Revista internacional de filosofía 
to. En el silogismo lo determinado no es separable del concepto que lo penetra. No se trata de una existencia a la que se le yuxtapone un concepto, sino de la realización -Entwicklung - objetiva de un concepto. Pero además el concepto determina la función de cada objeto en el organismo, y esto es expresado en el silogismo de silogismos.

Los dos primeros silogismos conclusivos de la Enciclopedia son denominados por Hegel, silogismos de la apariencia. Ellos exponen manifestaciones de la Idea. El tercer silogismo, en cambio, es denominado el silogismo de la idea de filosofía. Se trata del silogismo "Espíritu-Lógica-Naturaleza». Entre las dos ciencias reales se encuentra la ciencia de la Lógica como término medio. Surge, en el último parágrafo de la Enciclopedia la misma Idea absoluta, «la razón que se sabe, lo universal absoluto, como [término] medio suyo que se escinde en espíritu y naturaleza.» ${ }^{41}$ Pero esta razón se sabe en las dos manifestaciones de su actividad. Como actividad subjetiva, la razón da lugar a la esfera del espíritu. Como actividad sustancial a la de la naturaleza. Así, la naturaleza y el espíritu aparece como el juicio, el desdoblarse, la partición de la Idea. Naturaleza y espíritu son comprendidos desde el tercer silogismo como las manifestaciones de la razón. Es así como las ciencias filosóficas forman una sola ciencia, la única ciencia, la filosofía.

JuAn. J. PADial Benticuaga es profesor contratado doctor en la Universidad de Málaga,

Líneas de investigación:

Idealismo alemán, con especial dedicación a Hegel, antropología filosófica y Leonardo Polo.

Publicaciones recientes:

«Actos y espontaneidad cognoscitiva en Kant», Studia Poliana 16 (2014), 47-59.

«Venganza, realización de la libertad y guerra privada», Thémata 48 (2013), 85-93.

Dirección electrónica: jjpadial@uma.es

41 Hegel, G.W.F., Enz., § 577.

Suplemento 19 (2014) de Contrastes. Revista internacional de filosofía 